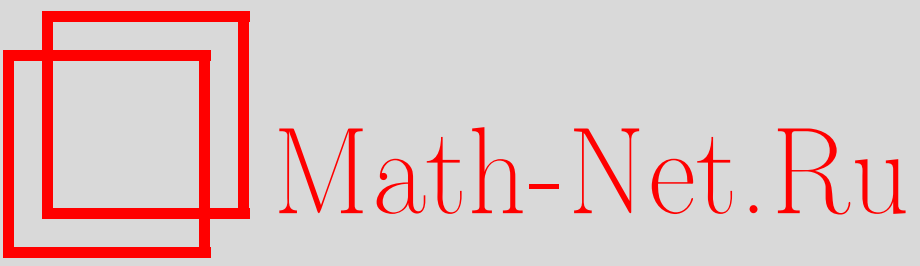

А. Н. Колмогоров, Почти горизонтальная турбулентность, УМН, 2004, том 59, выпуск 2, 3-8

DOI: https://doi.org/10.4213/rm714

Использование Общероссийского математического портала Math-Net.Ru подразумевает, что вы прочитали и согласны с пользовательским соглашением

http://www.mathnet.ru/rus/agreement

Параметры загрузки:

IP : 44.207 .124 .84

26 апреля 2023 г., 13:08:16 


\title{
ПОЧТИ ГОРИЗОНТАЛЬНАЯ ТУРБУЛЕНТНОСТЬ
}

\author{
А. Н. Колмогоров
}

1. Математическая модель “двумерной турбулентности” реально применяется к движению слоев некоторой конечной толшины. Предполагается, например, что такого рода двумерные рассмотрения приводят к разумной модели образования на течении, охватывающем слой в несколько сотен метров, меандров с горизонтальными размерами в сотни километров и их распадения на вихри, постепенно измельчаюшиеся до размеров нескольких километров. Если иметь в виду слой постоянной плотности с интенсивньм вертикальным перемешиванием, способньй довольно свободно скользить по подстилаюшему слою с сильной устойчивой стратификацией, то такие представления кажутся достаточно обоснованньми.

Но постепенно накопившиеся данные о детальной структуре вертикальных профилей температуры, солености, плотности и скорости в слоях с устойчивой стратификацией вместе с данными о турбулизированных "блинах" и разделяющих их не турбулизированных (в смысле трехмерной турбулентности малых масштабов) прослоек заставляют предполагать, что в этом случае в соответствуюших масштабах горизонтальные перемещения сравнительно тонких слоев в значительной мере независимы друг от друга. Должна возникать “почти горизонтальная турбулентность”, в кото-

Статья печатается без изменений. Рукопись А.Н. Колмогорова, по-видимому, была подготовлена во время его участия в одной из экспедиций “Дм. Менделеева” в Атлантический океан (1969 г.) и по кругосветному маршруту (1971 г.), организованными Институтом океанологии, руководимым в то время А.С. Мониным. Выбор темы, как пишет сам А.Н., стимулирован наблюдениями "на течении, охватьвающими несколько сот метров, меандров с горизонтальньми размерами в сотни километров и их распадением на вихри, постепенно измельчающиеся до размеров нескольких километров". В современной терминологии работа посвящена проблеме интенсивного перемешивания в пикноклинах - тонких слоях стратифицированной жидкости из-за внутренних волн с частотами, меньшими частоты Брента-Вяйсяля. Я бы отметил здесь два обстоятельства. Во-первых, традиционную для А.Н. научную проницательность, поскольку такой подход впоследствии действительно нашел отражение во многочисленных публикациях (см., например, монографию В. С. Модевич, В.И. Никулин, А.Г. Стеценко “Динамика внутреннего перемешивания в стратифицированной среде”, Институт гидромеханики УАН, Киев, Наукова думка, 1988). Во-вторых, и на мой взгляд это самое главное, подлинную любознательность и широту мшшления великого мыслителя, которьй занимался не толшко заоблачными абстрактными математическими построениями, но и с большим интересом спускался на землю для решения конкретных прикладных проблем. (Прим. Ф.В. Должанского.) 
рой структурные функции

$$
D_{i j}(\Delta \vec{x})=\overline{\Delta v_{i} \Delta v_{j}}
$$

растут при смешении по вертикали значительно быстрее, чем при смещении по горизонтали.

В этой заметке делается попытка создать предпосылки для разработки теории такой "почти горизонтальной турбулентности". Предлагается система упрощенных уравнений “почти горизонтальных движений". Отмечается, что эти уравнения обладают аффинной инвариантностью: вертикальный и горизонтальный масштаб можно менять независимо. Показьвается, что сделанное упрощение не мешает получить правильную теорию внутренних волн с частотами существенно меньшими, чем частота Брента-Вяйсяля.

Предлагаемая упрощенная система уравнений имеет вид

$$
\begin{gathered}
\frac{d u}{d t}+f v+\frac{\partial p}{\partial x}=\Phi_{x} \\
\frac{d v}{d t}-f v+\frac{\partial p}{\partial y}=\Phi_{y} \\
\frac{\partial p}{\partial z}-b=0 \\
\frac{\partial u}{\partial x}+\frac{\partial v}{\partial y}+\frac{\partial w}{\partial z}=0 \\
\frac{d b}{d t}=\Phi b .
\end{gathered}
$$

Как обычно, здесь $p$ - отклонение давления от стандартного $P=-z$ (стандартная плотность $\rho_{0}$ принята равной единице), $b$ - “плавучесть". Все величины $u, v, w, p$ и $b$ входят в уравнения в виде их средних по объему, делающему приемлемьм предположение "почти горизонтальности" (изгнание члена $\frac{d w}{d t}$ из (1.3) и включение влияния вязкости и диффузии в члены $\Phi_{x}, \Phi_{y}, \Phi_{b}$, имеюшие смысл взаимодействия с "микрокомпонентой" наших величин).

Система уравнений (1.1)-(1.5) инвариантна по отношению к замене переменных

$$
\begin{gathered}
x=\lambda x^{\prime}, \quad y=\lambda y^{\prime}, \quad u=\lambda u^{\prime}, \quad v=\lambda v^{\prime}, \quad p=\lambda^{2} p^{\prime}, \\
z=\lambda_{1} z^{\prime}, \quad w=\lambda_{1} w^{\prime}, \quad b=\lambda^{2} \lambda_{1}^{-1} b^{\prime},
\end{gathered}
$$

где $\lambda$ и $\lambda_{1}$ выбираются независимо друг от друга. Полезно заметить, что при такой замене не меняется и число Ричардсона

$$
\mathrm{Ri}=-\frac{\partial b}{\partial z} /\left(\frac{\partial u}{\partial z}\right)^{2}
$$

2. Для контроля характера сделанных упрощений построим соответствуюшую линейную теорию внутренних волн. Разобьем $p$ и $b$ на средние значения $\bar{p}$ и $\bar{b}$, зависяшие 
только от $z$, и отклонения от средних $p^{\prime}$ и $b^{\prime}$ и напишем линеаризованную систему уравнений для $u, w, p^{\prime}, b^{\prime}$ при движении в плоскости $(x, y)$ :

$$
\begin{aligned}
& L_{1}=\frac{\partial u^{\prime}}{\partial t}+\frac{\partial p^{\prime}}{\partial x}=0, \\
& L_{2}=\frac{\partial p^{\prime}}{\partial z}-b^{\prime}=0, \\
& L_{3}=\frac{\partial u^{\prime}}{\partial x}+\frac{\partial w^{\prime}}{\partial z}=0, \\
& L_{4}=\frac{\partial b^{\prime}}{\partial t}+w^{\prime} \frac{\partial \bar{b}}{\partial z}=0 .
\end{aligned}
$$

Из (2.1)-(2.5) получаем

$$
\frac{\partial^{3}}{\partial t \partial x \partial z} L_{1}-\frac{\partial^{3}}{\partial t \partial x^{2}} L_{2}+\frac{\partial^{3}}{\partial t^{2} \partial z} L_{3}-\frac{\partial^{2}}{\partial x^{2}} L_{4}=\frac{\partial^{4} w}{\partial t^{2} \partial z^{2}}-\frac{\partial \bar{b}}{\partial z} \frac{\partial^{2} w}{\partial x^{2}}=0
$$

или, положив $-\frac{\partial \bar{b}}{\partial z}=N^{2}$,

$$
\frac{\partial^{4} w}{\partial t^{2} \partial z^{2}}+N^{2} \frac{\partial^{2} w}{\partial x^{2}}=0
$$

Это уравнение отличается от полного уравнения

$$
\frac{\partial^{2}}{\partial t^{2}}\left(\frac{\partial^{2} w}{\partial x^{2}}+\frac{\partial^{2} w}{\partial z^{2}}\right)+N^{2} \frac{\partial^{2} w}{\partial x^{2}}=0
$$

отсутствием члена $\frac{\partial^{2} w}{\partial x^{2}}$, которьй для “почти плоских” волн мал по сравнению с членом $\frac{\partial^{2} w}{\partial z^{2}}$.

Полагая

$$
w=\alpha(z) \operatorname{Exp} i(k x-n t)
$$

получим

$$
n^{2} \alpha^{\prime \prime}+N^{2} k^{2} \alpha=0
$$

При изучении многослойной почти плоской турбулентности могут иметь значение волны старших мод, для понимания устройства которых рассматривают возмушения вида

$$
w=\gamma \operatorname{Exp} i(k x+m z-n t)=\gamma E,
$$

для которых в силу уравнений (2)

$$
\begin{aligned}
& w_{z}=i m \gamma E, \quad u_{x}=-i m \gamma E, \quad u=\frac{m}{k} \gamma E, \\
& u_{t}=-i \frac{n m}{k} \gamma E, \quad w_{t}=-i n \gamma E, \quad w_{x}=i k \gamma E \text {. }
\end{aligned}
$$

Дисперсионное соотношение у нас имеет простой вид

$$
n m=N k \text {. }
$$


“Почти плоский” характер волн требует, чтобы

$$
k \ll m, \quad n \ll N,
$$

что и объясняет законность упрощения (6) по сравнению с обычным

$$
n m=\sqrt{N^{2}-n^{2}} k .
$$

Рассмотрим еше условия законности линеаризации уравнений. Для этого, пользуясь (5) и (5.1) выпишем порядки величины отдельных членов полных производных $\frac{d u}{d t}$ :

$$
\left|\begin{array}{c|c|c}
\frac{\partial u}{\partial t} & u \frac{\partial u}{\partial x} & w \frac{\partial u}{\partial z} \\
\frac{n m}{k} \gamma & \frac{m^{2}}{k} \gamma & \frac{m^{2}}{k} \gamma
\end{array}\right|
$$

Мы видим, что нелинейные члены малы по сравнению с $\frac{\partial u}{\partial t}$, если

$$
m \gamma \ll n \text {. }
$$

Воспользовавшись тем, что амплитуда вертикальных перемешений имеет порядок $A \sim \frac{\gamma}{n},(6)$, мы можем написать $(7.1)$ в виде

$$
A \ll L_{z}=\frac{2 \pi}{m}
$$

Это, впрочем, вполне естественно: роль нелинейных эффектов зависит не от “крутизны” волн, а от отношения амплитуды вертикальных смещений к $L_{z}$.

Представлялось бы весьма интересным изучение "пологих", но существенно нелинейных волн. Не знаю, сильно ли поможет при этом упрощение уравнений, связанное с нашей "почти горизонтальностью” движений. Однако при отсутствии математической теории для обобшения эмпирических данных кажется весьма сушественньм замечание об аффинной инвариантности уравнений, позволяющей, например, предполагать, что при наличии того или иного механизма возрастания энергии волнового движения “опрокидывание” волн может в случае малости $L_{z} / L_{x}$ приобретать характер "набеганий” друг на друга весьма тонких слоев.

3. Предполагая, что $v=w=0, u, p$ и $b$ зависят только от $y$ и $z$ и пренебрегая членами $\Phi_{x}, \Phi_{y}, \Phi_{b}$, получим из (1) обычные уравнения геострофического потока

$$
\begin{gathered}
-f u+\frac{\partial p}{\partial y}=0 \\
\frac{\partial p}{\partial z}-b=0
\end{gathered}
$$

с не менее известным следствием

$$
f \frac{\partial u}{\partial z}=\frac{\partial b}{\partial y}
$$


Число Ричардсона приобретает вид

$$
\mathrm{Ri}=-f^{2} \frac{\partial b}{\partial z} /\left(\frac{\partial b}{\partial y}\right)^{2}
$$

При изучении многослойной турбулентности следует учитьвать наличие естественного отношения горизонтальных и вертикальных масштабов

$$
L_{x} / L_{z}=\frac{\partial b}{\partial x} / \frac{\partial b}{\partial y}
$$

которое обычно очень велико.

Пока мне трудно сказать, будет ли плодотворным путь построения на этой основе несколько умозрительной концепции локально однородной и изотропной по горизонтали почти плоской турбулентности. Но попытаться идти по этому пути, вероятно, следует.

4. Напишем уравнения для малых возмушений геострофического потока. ${ }^{1}$

Волны с учетом $f$. Дисперсионное соотношение имеет вид:

$$
D=\left|\begin{array}{cccc}
-i n & -f 0 & k / m & \\
+f & -i n & 0 & 0 \\
i k & 0 & i m & 0 \\
0 & 0 & -N^{2} & -i n
\end{array}\right|=-\left(n^{2}-f^{2}\right) n m+N^{2} n \frac{k^{2}}{m}=0
$$

откуда мы получаем:

$$
\begin{aligned}
& n^{2}=f^{2}+N^{2} \frac{k^{2}}{m^{2}}, \\
& -f a+i n b=0, \quad c_{k p}=\frac{n}{k}=\sqrt{\frac{f^{2}}{k^{2}}+\frac{N^{2}}{m^{2}}} \\
& i k a+i m c=0 \\
& -N^{2} c+i n d=0, \\
& \quad|a|=\frac{m}{k}|c|, \quad|b|=\frac{f}{n}|a|=\frac{f m}{n k}|c| .
\end{aligned}
$$

5. При развитии рассмотренных вьше возмушений должно возникать “многослойное меандрирование", которое, конечно, не подчиняется уже линейной теории. Возможно, что иногда действует механизм измельчения возникших меандров, предсказываемьй той или иной концепии “горизонтальной турбулентности" с передачей энергии, или вихря от одних масштабов к другим. Но естественно думать, что большую роль должно играть и взаимодействие слоев, расположенных друг над другом. При известных условиях, даже при большом числе Ричардсона среднего потока, могут возникать трехмерно турбулизированные "блины”.

\footnotetext{
${ }^{1}$ Ниже приводятся, к сожалению, незаконченные оригинальные вычисления А.Н. Колмогорова.
} 
Очередной не очень трудной задачей является расчет возмушений по линейной теории в случае $\frac{\partial \bar{b}}{\partial y} \neq 0$. Если устойчивая стратификация достигается при разных значениях поперечной координаты $y$ за счет разной комбинации температуры и солености, то при тонкослойном меандрировании должны возникнуть эффекты, которые последнее время привлекли столь большое внимание.

Для создания качественных представлений об этих процессах некоторую роль должны играть соображения об указанной аффинной инвариантности уравнений почти плоских движений. Они, однако, теряют применимость при возникновении турбулентного вертикального обмена между тонкими слоями и в масштабах, в которых число Рейнольдса тонких слоев уже нельзя считать несущественньм Поэтому постулированньй в самом начале масштаб осреднения в разной обстановке надо считать различным. Временные масштабы применимости развитой конщепции тоже должны быть, как мы видели, достаточно большими: при упрошении мы потеряли для внутренних волн ограничение $n<N$.

Поступила в редакцию

01.08 .2003 